[RAdiocarbon, Vol. 11, No. 1, 1969, P. 188-193]

\title{
TATA INSTITUTE RADIOCARBON DATE LIST VI
}

\author{
D. P. AGRAWAL and SHEELA KUSUMGAR \\ Tata Institute of Fundamental Research \\ Homi Bhabha Road, Colaba, Bombay-5
}

This date list is comprised of archaeologic and geophysical samples. The latter are in continuation of our investigations of bomb-produced radiocarbon in atmospheric carbon dioxide reported in Tata V. We continue to count samples in the form of methane; the techniques used have been described elsewhere (Agrawal et al., 1965).

Radiocarbon dates presented below are based on $\mathrm{C}^{14}$ half-life value of 5568 vr. For conversion to A.D./B.C. scale, 1950 A.D. has been used as base vr. Our modern reference standard is $95 \%$ activity of N.B.S. oxalic acid.

\section{GENERAL COMMENT}

Radiocarbon dating in India has been mainly confined to Neolithic and Chalcolithic cultures. Despite the dearth of datable material, attempts at evolving an absolute chronology for Stone-age cultures have now been started. Bone and shell samples were measured for some Microlithic cultures. Rock-shelters of Uttar Pradesh were dated ca. 2400 B.c. (TF-419). Adamgarh rock-shelters were dated ca. 5500 B.c. (TF-120, Radiocarbon, 1968, v. 10, p. 131). Kayatha culture of Madhya Pradesh appears to date from ca. 2000 B.c. Chirand Black-and-Red ware date (TF444) confirms the earlier dates (Radiocarbon, 1966, v. 8, p. 442). Terdal Neolithic culture was dated ca. 1800 B.c. A megalith from Halingali was dated ca. 100 B.c. Brief summaries of these excavations are available in Ghosh (1961-1966).

\section{ACKNOWLEDGMENTS}

The authors are thankful to Prof. D. Lal for his guidance, to S. K. Gupta for his help in preparing the manuscript, and to S. V. Kerkar for assistance.

\section{SAMPLE DESCRIPTIONS}

\section{ARCHAEOLOGIC SAMPLES}

TF-415. Atranjikhera, India, Black-and-Red ware deposits

$2450 \pm 200$ 500 B.C.

Charcoal from Atranjikhera (27 $42^{\prime} \mathrm{N}$ Lat, $78^{\circ} 44^{\prime} \mathrm{E}$ Long), Dist. Etah, baulk between Trenches $\mathrm{E}_{1}$ and $\mathrm{E}_{2}$, Layer 8 , depth $0.5 \mathrm{~m}$, Field No.3/1965. Sample subm. by R. C. Gaur, Aligarh Univ., Aligarh. Comment: sample is from baulk, hence stratigraphy may be poor. The large errors arise due to insufficiency of datable material. 


\section{TF-367. Chandraketugarh, India, historical deposits}

Shells from Chandraketugarh Dist. 24-Paraganas, Locus H2, depth 2.6 m, sample No. 2; sample subm. by C. R. Choudhury, Asutosh Mus., Calcutta-14. Comment: only inorganic fraction of shells was dated; organic fraction was not sufficient.

\section{Chirand series, Bihar}

Chirand $\left(25^{\circ} 45^{\prime} \mathrm{N}\right.$ Lat, $84^{\circ} 45^{\prime} \mathrm{E}$ Long), Dist. Saran, was excavated by B. S. Verma. Samples subm. by Dir. Archaeol. and Mus., Patna.

\section{TF-444. Black-and-Red ware deposits}

$2590 \pm 105$

Charred wood from Trench CRD-VII, Locus A2, Layer 16, depth $10.5 \mathrm{~m}$. NaOH pretreatment was also given.

\section{TF-445. Pit with microliths}

$$
3500 \pm 100
$$

Wood from Trench CRD-X, Locus A2, pit sealed by Layer 11, depth $8.2 \mathrm{~m}$. NaOH pretreatment was also given. Comment: sample is from pit in natural soil which yields large number of microliths and is sealed by Black-and-Red ware deposits; could be earlier.

\section{TF.445. Northern Black Polished ware $1930 \pm 105$ $\begin{array}{ll}\text { deposits } & \text { A.D. } 20\end{array}$}

Charred rice from Trench CRD-1c, Locus $B^{\prime}-C^{\prime}$, Layer 6, depth $3.25 \mathrm{~m}$. NaOH pretreatment was also given. Comment: sample derives from top of Northern Black Polished ware deposits.

\section{TF-685. Halingali, India, Megalithic deposits}

$$
1970 \pm 95
$$

Charcoal from Halingali (10 $0^{\circ} 0^{\prime} 30^{\prime \prime} \mathrm{N}$ Lat, $75^{\circ} 6^{\prime} 40^{\prime \prime}$ E I or Bijapur, Locus HGL Mo III, depth 1.31 to 1.4 m Fis. MgIII-24. $\mathrm{NaOH}$ pretreatment was also given Sampl Sankalia, Deccan College, Poona. Comment: date is younger than D. pected.

\section{Kayatha series, Madhya Pradesh}

Kayatha (23 $30^{\prime} \mathrm{N}$ Lat, $76^{\circ} \mathrm{E}$ Long), Dist. Ujjain was excavated by V. S. Wakankar, Madhav College, Ujjain, who subm. samples. $\mathrm{NaOH}$ pretreatment was given to all samples.

\section{TF.398. Chalcolithic deposits}

Charcoal from burnt Chalcolithic house, Trench KTH 1, Iayer 85 , depth $8.5 \mathrm{~m}$, Field No. 7 .

TF.405. Chalcolithic deposits

Charcoal, Trench KTH2, Layer 10, depth 3.5 m, Field No. 14. 


\section{TF-674. Northern Black Polished ware}

Charcoal, from Trench KTH4, Layer 11, depth not given, Field No.15. Comment: P.G. ware was also assoc.

TF-676. Chalcolithic deposits

Burnt wheat grains from Trench KTH4, Layer 14, depth not given, Field No. 17.

\section{TF-678. Chalcolithic deposits}

$3530 \pm 100$

Charcoal from Trench KTH4, Layer 18, Field No.19.

\section{TF-679. Chalcolithic deposits}

Charcoal from Trench KTH3, Layer 9, depth not given, Field No. 20.

\section{B.c.}

$3850 \pm 95$

TF-680. Kayatha culture

1900 B.C. No. 21.

Charcoal from Trench KTH3, Layer 13, depth not given, Field

\section{TF-586. Hallur, India, Neolithic period}

$3055 \pm 95$

1105 B.c.

Charcoal from Hallur ( $14^{\circ} 20^{\prime} \mathrm{N}$ Lat, $75^{\circ} 37^{\prime} \mathrm{E}$ Long), Dist. Dharwar, Trench 2, pit sealed by Layer 8 , depth $2.8 \mathrm{~m}$. Sample subm. by M. S. Nagaraj Rao, Karnatak Univ., Dharwar.

\section{TF-599. Kalibangan, India, bones}

$$
1930 \pm 100
$$

Animal bones from Kalibangan $\left(29^{\circ} 25^{\prime} \mathrm{N}\right.$ Lat, $74^{\circ} 05^{\prime} \mathrm{E}$ Long), Dist. Ganganagar, Locus XA-17 Od.1, Layer 4, depth $0.7 \mathrm{~m}$, Field No. KLB-2, XA-17 Qd.1/c/1965-66-1. Sample subm. by A. Ghosh. Comment: $\mathrm{C}^{14}$ date was obtained only from inorganic fraction and shows extent to which this fraction can be contaminated.

\section{Lekhahia series, Uttar Pradesh}

Lekhahia rock-shelters $\left(27^{\circ} 80^{\prime} 5^{\prime \prime} \mathrm{N}\right.$ Lat, $82^{\circ} 32^{\prime} \mathrm{E}$ Long), Dist. Mirzapur, were excavated by V. D. Misra. Samples subm. by G. R. Sharma, Allahabad Univ., Allahabad. There is no archaeologic date estimate available, but assoc. of aceramic geometric and non-geometric microliths indicates that $\mathrm{C}^{14}$ dates could be younger because only inorganic fraction of bones was dated.

TF-417. Rock-shelter skeleton

$3560 \pm 105$

Bones from Rock-shelter I, Skeleton No. VI, Locus 5-7, Layer 4, depth 0.15 m, Field No. LKH(M)RS-1/64/B-2. 
TF-419. Rock-shelter skeleton

$4240 \pm 110$

2290 B.c.

depth m, Field No. LKH(M)RS-1/64/B-4.

TF-744. Langhnaj, India, Microlithic culture

$3875 \pm 105$

1925 B.C.

Bones from Langhnaj (23 $27^{\prime}$ E Lat, $72^{\circ} 32^{\prime}$ E Long), Dist. Mehsana, Trench BFGC, depth 1.2 to $1.8 \mathrm{~m}$. Sample subm. by H. D. Sankalia. Comment: different bones samples R. Nos. 3828, 3921, 4707, 4326, and 5188 from lower and middle levels were mixed for $\mathrm{C}^{14}$ dating. Only inorganic fraction was dated; contamination probability is high.

\section{Rajbadidanga series, W Bengal}

Rajbadidanga $\left(24^{\circ} 01^{\prime} \mathrm{N}\right.$ Lat, $88^{\circ} 11^{\prime} \mathrm{E}$ Long), Dist. Murshidabad, was excavated by S. R. Das, Dept. of Archaeol., Univ. of Calcutta, who subm. samples.

TF-629. Historical deposits

$1495 \pm 90$

Comment: assoc. finds have terracotta seals.

TF-634. Historical deposits

$1520 \pm 90$

Charcoal from Trench B5, Layer 8, depth $3.1 \mathrm{~m}$, Field No. 9 .

TF-681. Tripuri, India, Early Historic period

$2155 \pm 100$

205 B.c.

Charcoal from Tripuri (23 $8^{\prime} \mathrm{N}$ Lat, $79^{\circ} 50^{\prime} \mathrm{E}$ Long), Dist. Jabalpur, Locus II, Layer 5, depth $2.8 \mathrm{~m}$, Field No. 246. Sample subm. by H. D. Sankalia. Rootlets were handpicked. $\mathrm{NaOH}$ pretreatment was also given.

\section{TF-689. Palmleaf manuscript, India}

Sample subm. by K. S. Karanth, Puttor, Mysore.

$$
40 \pm 95
$$

\section{A.D. 1910}

\section{Terdal series, Mysore}

Terdal ( $16^{\circ} \mathrm{N}$ Lat, $75^{\circ} 5^{\prime} \mathrm{E}$ Long), Dist. Bijapur. Site is being excavated by A. Sundara, Deccan College, Poona. Samples subm. by H. D. Sankalia.

TF-683. Neolithic culture

$3615 \pm 120$

Charcoal from Trench 1, Layer 3 depth 45 to 1665 B.c.

\section{TF-684. Neolithic culture} $3775 \pm 95$

Charcoal from Trench 1, Layer 2, depth .17 to $.25 \mathrm{~m}$, Field 


\section{Ujjain series, Madhya Pradesh}

Ujjain (23 $11^{\prime} \mathrm{N}$ Lat, $75^{\circ} 46^{\prime} \mathrm{E}$ Long), Dist. Ujjain, samples subm. by A. Ghosh, site excavated by N. R. Banerjee.

\section{TF-407. Black-and-Red ware deposits}

$$
1990 \pm 100
$$

40 B.C. 108.

Charcoal, Locus O-I, Layer 23, depth 4.4 m, Field No. UJN 17 Ext/

$\begin{array}{cc}2335 & \pm 95 \\ 385 & \text { B.c. }\end{array}$ 155.

Charcoal, Locus IV-V, Layer 28, depth 5.50 m, Field No. U JN 28/

\section{GEOPHYSICAL SAMPLES}

\section{$C^{14}$ in Atmospheric Carbon Dioxide}

Atmospheric $\mathrm{CO}_{2}$ samples were coll. at 3 stations: 1) Gulmarg (34 ${ }^{\circ}$ 04' N Lat, 74 25' E Long), alt. $2745 \mathrm{~m}$; 2) Khandala (18 $45^{\prime} \mathrm{N}$ Lat, $73^{\circ}$ $22^{\prime}$ E Long), alt. $550 \mathrm{~m}$; 3) Kodaikanal (10 $15^{\prime} \mathrm{N}$ Lat, $77^{\circ} 31^{\prime} \mathrm{E}$ Long), alt. $2300 \mathrm{~m}$. Gulmarg and Kodaikanal series samples are continuation of data published in Tata V. Khandala was new sta., at about same lat. as Bombay, but far away from industrial area.

The following tables give time of exposure of $0.1 \mathrm{~N} \mathrm{NaOH}$ (carbonate-free) solution to air and observed $\%$ excess of $\mathrm{C}^{14}$ in atmospheric $\mathrm{CO}_{2}$ samples above reference level of age-corrected 1890 wood, i.e., 0.95 NBS oxalic acid.

\section{TABLE 1}

Atmospheric Radiocarbon Activity series, Gulmarg

\begin{tabular}{llcrc}
\hline Sample No. & \multicolumn{3}{c}{ Exposure Dates } & $\delta C^{14 \%}$ \\
\cline { 1 - 3 } TF-492 & June & 1, 1965-June 15, 1965 & $73.74 \pm 1.73$ \\
TF-497 & June 15, 1965-July 1, 1965 & $76.12 \pm 2.02$ \\
TF-498 & July 1, 1965-July 15, 1965 & $80.05 \pm 1.89$ \\
TF-499 & July 15, 1965-Aug. 1, 1965 & $72.59 \pm 1.74$ \\
TF-652 & Sept. 1, 1965-Sept. 10, 1965 & $71.99 \pm 1.79$ \\
TF-654 & Oct. 1, 1965-Oct. 10, 1965 & $73.59 \pm 1.79$ \\
\hline
\end{tabular}


TABLE 2

Atmospheric Radiocarbon Activity series, Khandala

\begin{tabular}{llcc}
\hline Sample No. & & Exposure Dates & $\delta C^{14 \%}$ \\
\hline TF-553 & Aug. 31, 1965-Sept. 10, 1965 & $68.72 \pm 1.77$ \\
TF-554 & Sept. 10, 1965-Sept. 20, 1965 & $70.78 \pm 1.88$ \\
TF-667 & Sept. 20, 1965-Sept. 30, 1965 & $69.25 \pm 1.77$ \\
TF-668 & Oct. 1, 1965-Oct. 10, 1965 & $69.93 \pm 1.74$ \\
TF-670 & Oct. 20, 1965-Oct. 30, 1965 & $73.11 \pm 1.81$ \\
\hline
\end{tabular}

TABLE 3

Atmospheric Radiocarbon Activity series, Kodaikanal

\begin{tabular}{llccc}
\hline Sample No. & & Exposure Dates & $\delta C^{14 \%} \%$ \\
\cline { 1 - 2 } & June & 1, 1965-June 16, 1965 & $73.06 \pm 1.77$ \\
TF-542 & July 1,1965 -July 16, 1965 & $70.55 \pm 1.75$ \\
TF-658 & Aug. 1, 1965-Aug. 10, 1965 & $68.40 \pm 1.74$ \\
TF-660 & Sept. 1, 1965-Sept. 10, 1965 & $70.71 \pm 1.70$ \\
TF-661 & Sept. 20, 1965-Oct. 1, 1965 & $70.51 \pm 1.87$ \\
TF-662 & Oct. 1, 1965-Oct. 10, 1965 & $73.00 \pm 1.80$ \\
TF-66t & Nov. 1, 1965-Nov. 10, 1965 & $71.83 \pm 1.76$ \\
\hline
\end{tabular}

Date lists:
Tata Institute IV
Agrawal and Kusumgar, 1966

REFERENCES

Agrawal, D. P., Kusumgar, S., and Lal, D., 1965, The measurement of $\mathrm{C}^{14}$ activity and some age determinations of archaeological samples: Current Sci., v. 34, p. 394-397. Agrawal, D. P. and Kusumgar, Sheela, 1966, Tata Institute radiocarbon date list IV: Radiocarbon, v. 8, p. 442-452.

p. 1968, Tata Institute radiocarbon date list V: Radiocarbon, v. 10, no. 1,

Ghosh, A., 1961-1966, Indian Archaeology-a Review: Archaeol. Survey of India. 